\title{
Selective triggering of magnetic flux avalanches by an edge indentation
}

\author{
Lu Jiang, ${ }^{1}$ Cun Xue, ${ }^{2, *}$ L. Burger, ${ }^{3}$ B. Vanderheyden, ${ }^{3}$ A. V. Silhanek, ${ }^{4}$ and You-He Zhou ${ }^{5,1, \dagger}$ \\ ${ }^{1}$ School of Aeronautics, Northwestern Polytechnical University, Xi'an 710072, China \\ ${ }^{2}$ School of Mechanics, Civil Engineering and Architecture, \\ Northwestern Polytechnical University, Xi'an 710072, China \\ ${ }^{3}$ SUPRATECS group, Montefiore Research Unit, \\ Department of Electrical Engineering and Computer Science, \\ Université de Liège, B-4000 Sart Tilman, Belgium \\ ${ }^{4}$ Experimental Physics of Nanostructured Materials, Q-MAT, \\ CESAM, Université de Liège, B-4000 Sart Tilman, Belgium \\ ${ }^{5}$ Key Laboratory of Mechanics on Disaster and Environment in Western China, Ministry of Education of China, \\ Department of Mechanics and Engineering Sciences, Lanzhou University, Lanzhou 730000, China
}

(Dated: April 23, 2020)

\begin{abstract}
We numerically investigate the effect of an edge indentation on the threshold field of thermomagnetic instabilities in superconducting films subjected to a ramping magnetic field, applied perpendicular to the plane of the film. In particuar, we are able to address the question on whether edge indentations promote magnetic flux avalanches. For magnetic field-independent critical current densities model, the triggering of the first magnetic flux avalanche systematically occurs at the edge indentation. In contrast to that, for the more realistic field-dependent critical current density model the first flux avalanche can take place either at or away from the indentation. This selective triggering of magnetic flux avalanches is shown to result from the variation of the threshold magnetic field for the first flux avalanche triggered at the indentation and the reduction of the critical current density by large local magnetic fields at the tip of the indentation which translates in a lower power density dissipated near the tip. We demonstrate that this interplay can be tuned by varying the indentation size, ramp rate of applied field $\dot{H}_{a}$, and working temperature $T_{0}$. We build up a phase diagram in the $\mu_{0} \dot{H}_{a}-T_{0}$ plane with well-defined boundaries separating three distinct regimes of thermomagnetic instability.
\end{abstract}

\section{INTRODUCTION}

Microscopic-scale defects in an ohmic conductor alter the flow of electric current in a region extending over a few times the size of the defect. In superconductors, microscopic defects lead to modifications of the current streamlines over a range much larger than the defect linear size and, therefore, produce a major impact on the response of the system [1]. When such defects lie at the edge of the superconducting sample, even more dramatic consequences can be expected, as a result of the penetration of magnetic flux through the sample borders.

More specifically, it is widely believed that magnetic flux avalanches of thermomagnetic origin[2-4] should preferably nucleate at the location of defects. The main argument behind this belief leans on the fact that edge indentations give rise to current crowding in their vicinity [5], i.e. a larger current density $\mathbf{J}$ and, hence, an enhanced local electric field $\mathbf{E}$, both conspiring to locally generate substantial Joule heating $Q=\mathbf{J} \cdot \mathbf{E}$. Thus, theoretically, magnetic flux avalanches are predicted to be larger and occur more frequently at the location of the defect [6-9]. This phenomenon was observed in $\mathrm{YBa}_{2} \mathrm{Cu}_{3} \mathrm{O}_{7-\delta}$ by Baziljevich et al [10].

Surprisingly, recent experimental evidence in Nb suggests that edge indentations might actually have the op-

\footnotetext{
*xuecun@nwpu.edu.cn

$\dagger$ zhouyh@lzu.edu.cn
}

posite effect, that is thermomagnetic avalanches are statistically less likely to occur in their vicinity [11]. This is illustrated by the set of magneto-optical images presented in Fig. 1, obtained in a $2 \times 2 \mathrm{~mm}^{2}$ square-shaped $140 \mathrm{~nm}$-thick $\mathrm{Nb}$ film with edge indentations which are $10 \mu \mathrm{m}$ wide and are sitting at opposite sides (upper and lower rows). More information about the technique of magneto-optical imaging (MOI) used to image flux density in various superconductors can also be seen in Refs. [12-20], including the smooth flux penetration [13-15] and the lighting-like flux avalanches (e.g. $\mathrm{Nb}[12,16]$, $\mathrm{MgB}_{2}[17,18]$ and $\left.\mathrm{YB}_{2} \mathrm{Cu}_{3} \mathrm{O}_{7-x}[19,20]\right)$. These images were taken at $T_{0}=2.5 \mathrm{~K}$ and in zero-field cooling. Bright (dark) color indicates high (low) local magnetic fields. Panels (a) and (b) show flux expulsion from the interior of the sample at $\mu_{0} H_{a}=0.22 \mathrm{mT}$ and magnetic flux concentration at the border. Note that the two opposite indentations are visible close to the center of each panel. At $\mu_{0} H_{a}=0.4 \mathrm{mT}$ (panels (c) and (d)) a clear plume-shaped magnetic flux penetration emanating from the indentations is observed. Further increasing the applied magnetic field to $\mu_{0} H_{a}=0.58 \mathrm{mT}$ leads to abrupt burst of flux avalanches, distinctly away from the indentation (panels (e) and (f)). Panel (g) shows a more spectacular multibranched avalanche avoiding the edge indentation, as observed in a sample with similar geometry and composition.

In this work, we shed light on the origin of the above described discrepancy between the theoretical expectation and the experimental findings. By performing nu- 

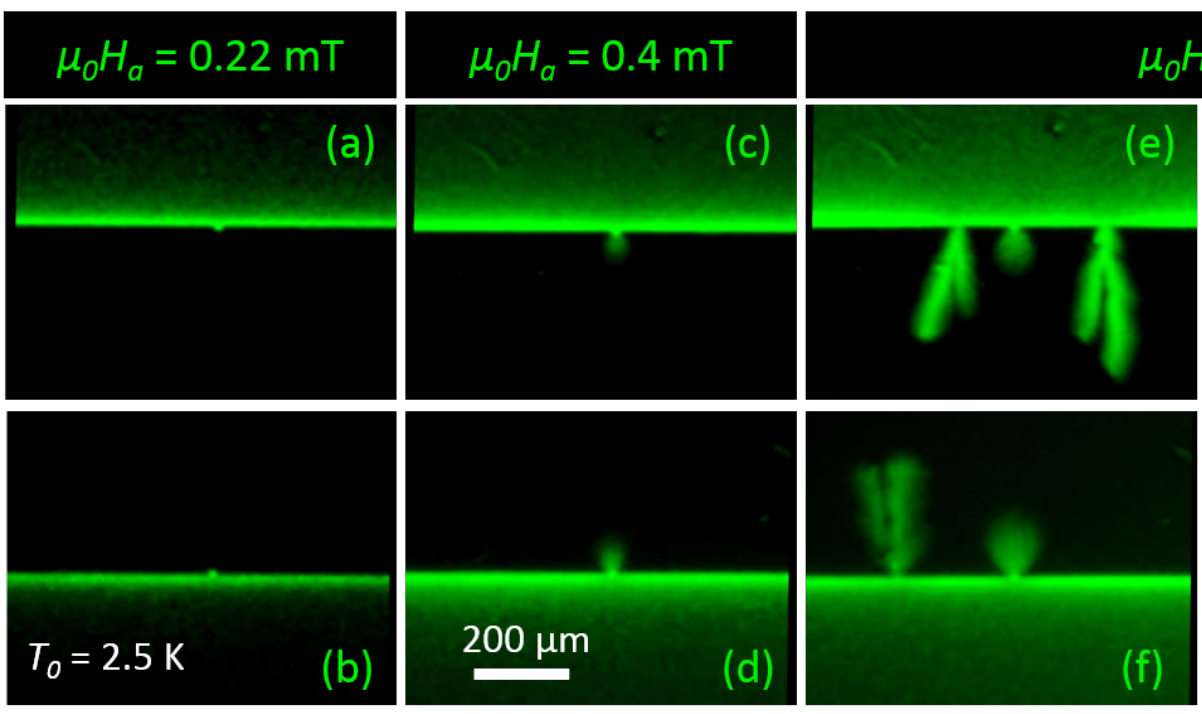

\section{$\mu_{0} H_{a}=0.58 \mathrm{mT}$}

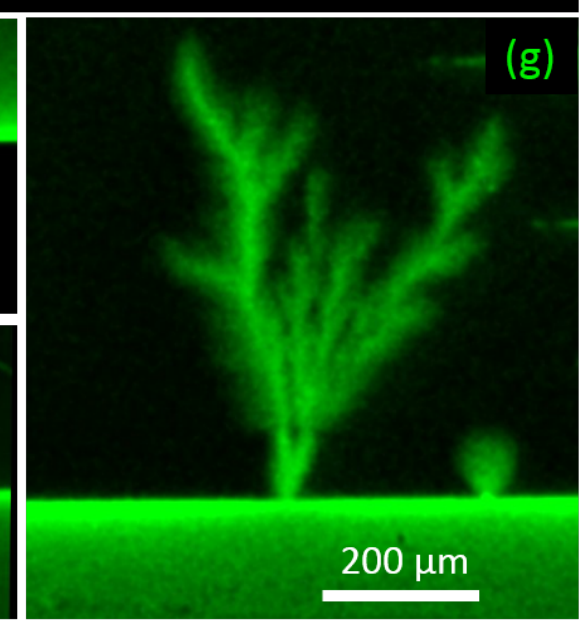

FIG. 1. Selected set of magneto-optical images obtained in a $2 \times 2 \mathrm{~mm}^{2}$ square-shaped $140 \mathrm{~nm}$-thick $\mathrm{Nb}$ film with $10 \mu \mathrm{m}$ wide triangular edge indentations sitting at opposite sides (upper and lower rows). All images were acquired at $T_{0}=2.5 \mathrm{~K}$ and in zero-field cooling. Bright (dark) color indicates high (low) local magnetic fields. These particular samples have been previously investigated in Ref. [21].

merical simulations of the thermomagnetic instabilities in a superconducting film with a field-dependent critical current density, we show the existence of three regimes, corresponding respectively to no avalanches, avalanches triggered at the indentation, and avalanches triggered away from the indentation. In addition, we investigate the effect of indentation size, working temperature and ramping rate of magnetic field on the selective triggering of magnetic flux avalanches. The paper is organized as follows: Section II introduces the theoretical model, Section III presents the numerical simulated results and discuss how edge indentations affect the flux penetration and the thermomagnetic stability of superconducting films. Finally, we summarize the most salient results is Section IV.

\section{MODEL}

We consider a square superconducting film $(2 a \times 2 a)$ of thickness $d$ subjected to a gradually increasing transverse field $H_{a}$, as schematically shown in Fig. 2. The superconducting film is assumed to be in thermal contact with a substrate which is kept at a constant temperature $T_{0}$. The superconducting film contains a triangular indentation of width $2 s$ and depth $s$ sitting at the middle of one of its edges.

The electrodynamics of the superconducting film is solved from Maxwell's equations in the quasistatic limit (the displacement current is neglected),

$$
\dot{\boldsymbol{B}}=-\nabla \times \boldsymbol{E}, \nabla \times \boldsymbol{H}=\boldsymbol{J} \delta(z) \text { and } \nabla \cdot \boldsymbol{B}=0,
$$

with $\boldsymbol{B}=\mu_{0} \boldsymbol{H}$ and $\nabla \cdot \boldsymbol{J}=0$. Here, the film thickness $d$

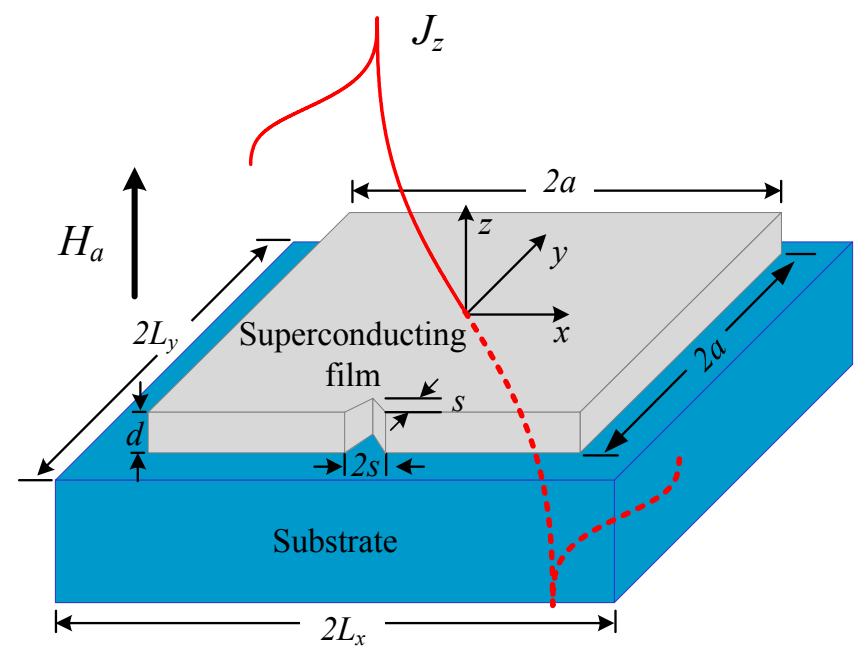

FIG. 2. Schematic of the sample. A square superconducting film of width $2 a$ and thickness $d$ with a triangular indentation is in thermal contact with a substrate. The substrate is kept at a constant temperature $T_{0}$. The heat exchange between substrate and superconducting film takes place with a heat transfer coefficient $h$. The red curve shows the distribution of the sheet current $J_{z}$ along the x-axis as obtained from the Kim model.

is neglected against the other characteristic length scales and the current density is given as $\boldsymbol{J} \delta(z)$, with $\boldsymbol{J}$ the sheet current and $\delta(z)$ the Dirac delta distribution.

The heat transport in the system is governed by the equation

$$
d c \dot{T}=d \kappa \nabla^{2} T-h\left(T-T_{0}\right)+\boldsymbol{J} \cdot \boldsymbol{E},
$$


where $\kappa, h$ and $c$ are the thermal conductivity of the superconducting film, the coefficient of heat transfer between the superconducting film and the substrate, and the specific heat of the film, respectively. The thermal parameters are assumed to be proportional to $T^{3}$, i.e., $\kappa=\kappa_{0}\left(T / T_{c}\right)^{3}, h=h_{0}\left(T / T_{c}\right)^{3}$ and $c=c_{0}\left(T / T_{c}\right)^{3}[22]$. The last term of Eq. (2), $\boldsymbol{J} \cdot \boldsymbol{E}$, represents Joule heating in the superconducting film.

The constitutive relationship between current and electric field is given as [22]

$$
\boldsymbol{E}=\rho(J) \boldsymbol{J} / d,
$$

with a resistivity law

$$
\rho(J)= \begin{cases}\rho_{0}\left(J / J_{c}\right)^{n-1}, & J \leq J_{c}, T \leq T_{c}, \\ \rho_{0}, & J>J_{c}, T \leq T_{c} \\ \rho_{n}, & T>T_{c}\end{cases}
$$

where $\rho_{0}$ is a constant resistivity, $\rho_{n}$ is the normal state resistivity, $J_{c}$ is the critical sheet current density, and $n$ is the flux creep exponent taken as $n=n_{0} T_{c} / T$, with $n_{0}$ constant. For $J \leq J_{c}$ and $T \leq T_{c}$, the $E$ - $J$ law is highly nonlinear and describes flux creep. In this regime, the superconducting film is typically divided in an outer region which is penetrated by the magnetic flux and a inner region which is flux-free. For $J>J_{c}$ and $T \leq T_{c}$, the $E$ - $J$ law is linear and describes flux flow. Last, for $T>T_{c}$, the material follows a linear Ohm's law with the normal state resistivity.

The critical current density is further assumed to depend on the magnetic flux density, following a field- and temperature-dependent critical current law [23],

$$
J_{c}=J_{c 0}\left(1-\frac{T}{T_{c}}\right) \frac{B_{0}}{|B|+B_{0}},
$$

where $J_{c 0}$ is the zero-field sheet current and $B_{0}$ is a constant field which represents the degree of field dependence. McDonald and Clem [24] derived the exact solutions for the flux penetration into a infinitely long superconducting film with such field-dependent critical current. Xue et al [25] extended it to the case where both a transport current and a magnetic field are applied to a superconducting film. In the case considered here, with such a field-dependence, the distribution of sheet curren$\mathrm{t}$ across the film typically has the shape illustrated in Fig. 2, with a decrease of $|\boldsymbol{J}|$ towards the edges [24].

Equations (1) are numerically solved using the integral method of Refs. [22, 26]. The sheet current density is derived from the local magnetization $g=g(x, y)$,

$$
\boldsymbol{J}=\nabla \times g \hat{\boldsymbol{z}},
$$

where $\hat{z}$ is the unit vector normal to the film plane. The perpendicular component of the magnetic field $B_{z}(\boldsymbol{r})$ is obtained from $g$ by means of Biot-Savart's law,

$$
B_{z}(\boldsymbol{r})-H_{a}=\int_{S} d^{2} r^{\prime} Q\left(\boldsymbol{r}, \boldsymbol{r}^{\prime}\right) g\left(\boldsymbol{r}^{\prime}\right),
$$

where the integral is carried over the superconducting film and $Q\left(\boldsymbol{r}, \boldsymbol{r}^{\prime}\right)$ is a 2-dimensional kernel satisfying $\mathcal{F}(Q)=k / 2[27]$, with $\mathcal{F}$ denoting the Fourier transform and $k=|\boldsymbol{k}|$, with $\boldsymbol{k}$ an in-plane wavevector. Equation (7) can be inverted to express the time derivative of $g$ as

$$
\dot{g}(\boldsymbol{r}, t)=\mathcal{F}^{-1}\left\{\frac{2}{k} \mathcal{F}\left[\dot{B}_{z}(\boldsymbol{r}, t) / \mu_{0}-\dot{H}_{a}(t)\right]\right\},
$$

where $\mathcal{F}^{-1}$ denotes the inverse Fourier transform. Inside the superconducting film, $\dot{B}_{z}$ is obtained from Faraday's law and the constitutive law of Eq. (4), as

$$
\dot{B}_{z}=\nabla \cdot(\rho \nabla g) / d .
$$

For the domain outside the superconducting film, $\dot{B}_{z}$ can be determined by an iterative method which guarantees that $\dot{g}=0$, hence precluding any current flow oustide the superconductor, as reported in [22].

Equation (8) is solved with the fast Fourier transform (FFT) [22] over a domain consisting of a rectangular area of size $2 L_{x} \times 2 L_{y}$ discretized on a $256 \times 256$ equidistant grid, where $L_{x}=L_{y}=1.3 a$. The superconducting film half-width and thickness are $a=1.0 \mathrm{~mm}$ and $d=100 \mathrm{~nm}$. The superconducting parameters are given as $T_{c}=9.2 \mathrm{~K}$, $j_{c 0}=J_{c 0} / d=1.2 \times 10^{11} \mathrm{~A} / \mathrm{m}^{2}$, and $\rho_{0}=\rho_{n}=5 \times 10^{-9}$ $\Omega \mathrm{m}[28]$. A random disorder is introduced by reducing $j_{c 0}$ by $10 \%$ at $5 \%$ of the grid points, selected randomly. The thermal parameters $\kappa_{0}, h_{0}$, and $c_{0}$, which correspond to $\kappa, h$, and $c$ at $T=T_{0}$, are given as $20 \mathrm{~W} / \mathrm{Km}, 10^{4}$ $\mathrm{W} / \mathrm{Km}^{2}$ and $3 \times 10^{4} \mathrm{~W} / \mathrm{Km}^{3}$, respectively [28]. We adop$\mathrm{t} n_{0}=20$ and limit the creep exponent to $n(T) \leq 100$ for convergence issues [29]. The superconducting film is exposed to an increasing magnetic field ramped at a rate $\dot{H}_{a}$, from an initial zero-field cooled state. The magnetic response of the film is determined from $T$ and $g$, by integrating Eq. (2) and Eq. (8) over time.

\section{RESULTS AND DISCUSSION}

The field dependence of the critical current density has been shown to influence both the magnetic flux penetration in superconducting films [11] and the occurence of thermomagnetic instabilities [30]. Our first goal is to discuss these effects when considering a thin superconducting film with a single triangular indentation of height $s=62 \mu \mathrm{m}$, subjected to a magnetic field ramped at a rate $\mu_{0} \dot{H}_{a}=3 \mathrm{~T} / \mathrm{s}$, with a fixed substrate temperature $T_{0}=2.5 \mathrm{~K}$. Figure 3 shows the calculated magnetic flux distributions in a superconducting film under either a low applied magnetic field of $1.5 \mathrm{mT}$ (left column), or a magnetic field equal to the threshold field $\mu_{0} H_{t h}$ required to trigger the first magnetic flux avalanche (right column). The distributions are shown for different parameter ratios $B_{0} / B_{f}=\infty(\mathrm{a}, \mathrm{b}), 5(\mathrm{c}, \mathrm{d})$, and $1(\mathrm{e}, \mathrm{f})$, where $B_{f}=\mu_{0} j_{c 0} d / \pi$.

As the applied magnetic field increases to $\mu_{0} H_{a}=1.5$ $\mathrm{mT}$, the magnetic flux gradually penetrates into the su- 

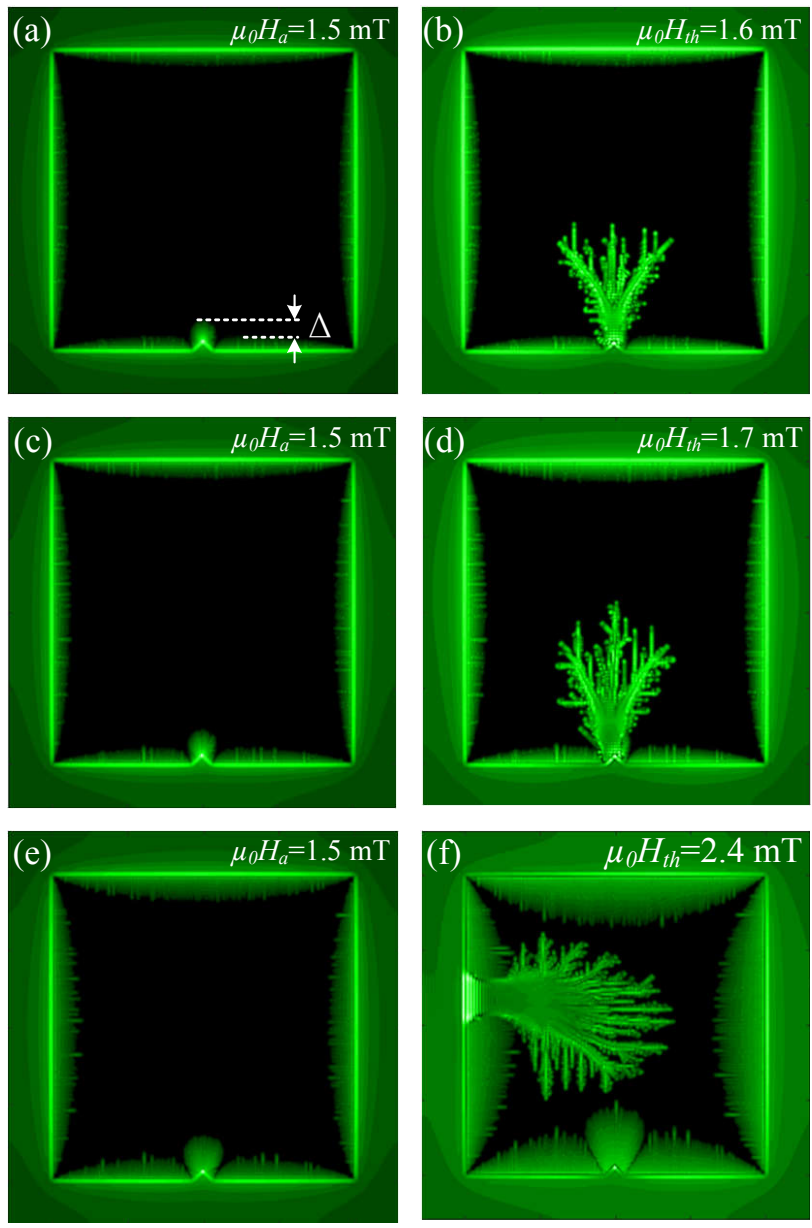

FIG. 3. Simulated distributions of $B_{z}$ in a superconducting film with a triangular indentation of size $s \times 2 s$ where $s=62 \mu \mathrm{m}$, for $\mu_{0} H_{a}=1.5 \mathrm{mT}[(\mathrm{a}),(\mathrm{c}),(\mathrm{e})]$ and for magnetic fields corresponding to the onset of avalanches [(b), (d), (f)]. The excess flux penetration depth $\Delta$ is indicated in panel (a). The results from the first row to the last one are obtained with $B_{0} / B_{f}=\infty, 5$, and 1 , respectively. The image brightness represents the magnitude of $B_{z}$, as in the experimental magneto-optical images. In all cases, the substrate temperature is $T_{0}=2.5 \mathrm{~K}$ and the ramp rate is $\mu_{0} \dot{H}_{a}=3 \mathrm{~T} / \mathrm{s}$.

perconducting film. The current density reaches its critical value in the flux-penetrated regions, while Meissner currents with $J<J_{c}$ flow in the non-penetrated ones. Figure 3 shows that the magnetic flux penetration is deeper near the tip of the indentation, by an amount $\Delta$ called the excess penetration depth [8]. According to the authors of Ref. [8], the excess depth $\Delta$ arises as the currents running parallel to the sample edge must locally circumvent the triangular defect. This leads to a crowding of the Meissner currents near the tip, where the sheet current reaches $J_{c}$, thus extending further the magnetic flux penetration depth. Direct inspection of the images presented in the left column of Figure 3 shows that $\Delta$ is larger when $J_{c}(B)$ decays more rapidly with $B$, i.e. for smaller ratios $B_{0} / B_{f}$. This result stems from a suppression of the critical sheet current $J_{c}$ by the local magnetic field, so that the Meissner sheet current reaches its critical value under smaller applied fields, allowing the flux front to penetrate deeper into the film.

The most interesting point is the influence of the ratio $B_{0} / B_{f}$ on the location of the nucleation of the first flux avalanche, as shown in Fig. 3(b), (d), and (f). For the limit $B_{0} \rightarrow \infty$ (Fig. 3(b)) the first flux avalanche nucleates at the tip of the indentation, for an applied flux density of $1.6 \mathrm{mT}$. This scenario agrees both with the observations of Baziljevich et al. [10] on a $\mathrm{YBa}_{2} \mathrm{Cu}_{3} \mathrm{O}_{7-\delta}$ film with a $0.5 \mathrm{~mm}$ long and $80 \mu \mathrm{m}$ wide slit and applying the magnetic field at a rate of $3000 \mathrm{~T} / \mathrm{s}$, as well as with the theoretical predictions reported by several authors in Ref. [6-9]. These predictions follow from the consideration that current crowding near the indentation induces an increase in the sheet current density and the strength of the electric field, both effects contributing to an increased Joule heating $\boldsymbol{J} \cdot \boldsymbol{E}$. This in turn favors the thermomagnetic instability which is at the origin of magnetic flux avalanches. However, the situation appears to be somewhat more complex with a $J_{c}(B)$ law. For $B_{0} / B_{f}=5$ (Fig. 3(d)), it can be observed that the first avalanche is still triggered at the indentation, under a magnetic flux density of $1.7 \mathrm{mT}$. For $B_{0} / B_{f}=1$ (Fig. 3(f)), on the other hand, the first avalanche nucleates away from the indentation, along a border and at a field of $2.4 \mathrm{mT}$. The preferential triggering of avalanches away from the defect was previously observed in $\mathrm{Nb}$ films $[11,21]$. This situation was interpreted as resulting from a reduction of surface barriers near the indentation, inducing a release of the magnetic flux pressure and a smooth flux penetration through the defect. The latter then acts as a magnetic flux faucet, whereas the magnetic pressure can still build-up away from the defect, until thermomagnetic instabilities with large releases of magnetic flux are triggered. Here, a similar effect may occur, now with the help of the field-dependent current density. Indeed, at the indentation the local magnetic field is the largest and therefore $J_{c}$ is locally depressed allowing further entrance of additional magnetic flux.

Independently of the nucleation loci of the first avalanche, Fig. 3 shows that the threshold applied magnetic flux density increases as $B_{0} / B_{f}$ is decreased. This result can be naturally accounted for within the thermomagnetic instability model presented in Ref. [31]. Fig. 4 shows generic curves for the threshold applied magnetic field as a function of $J_{c}$. The blue and red dashed lines in Fig. 4 are the threshold applied magnetic field$\mathrm{s}$ leading to the first avalanche triggered away from the indentation and at the indentation, respectively. It is assumed that the conditions for triggering avalanches away from the indentation are nearly invariable in a range of the parameter $B_{0} / B_{f}$. The presence of the indentation bends the current flowing around it, which increases the local magnetic field. As a consequence, the threshold magnetic field for which the first flux avalanche is trig- 


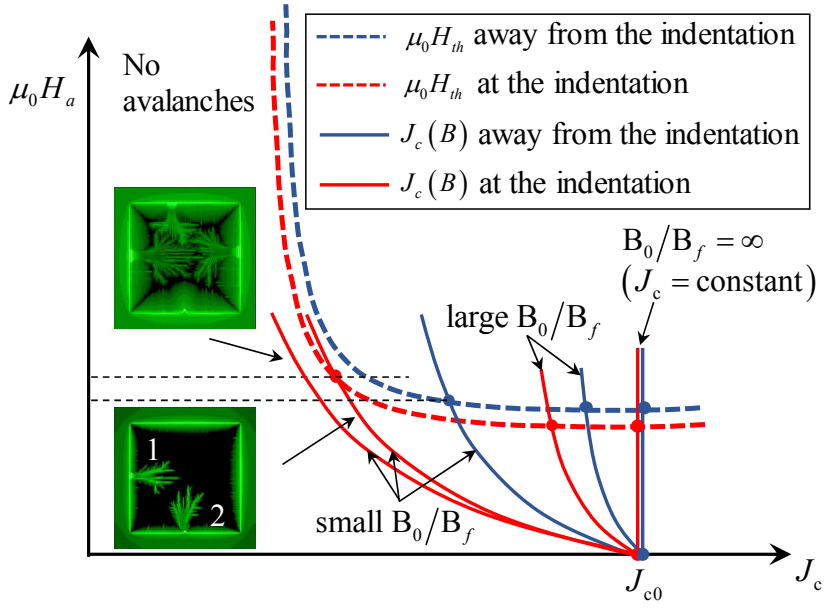

FIG. 4. Generic curves giving the threshold fields $\mu_{0} H_{\text {th }}$ away from the indentation and at the indentation as a function of $J_{c}$. Several cases of $J_{c}(B)$ laws with different $B_{0} / B_{f}$ are shown, illustrating the increase of $\mu_{0} H_{\text {th }}$ as $B_{0} / B_{f}$ is decreased.

gered at the indentation is lowered. Thus, the red dashed curve, showing this threshold field as a function of $J_{c}$, lies below the blue dashed curve, showing the threshold magnetic field for which the first flux avalanche is triggered away from the indentation. The red and blue solid lines represent the $J_{c}\left(H_{a}\right)$ model for different $B_{0} / B_{f}$. For a field-independent critical current density $J_{c}=J_{c 0}$ $\left(B_{0} / B_{f}=\infty\right)$, the threshold field is always reached first at the indentation as shown by the red spot at the intersection of the solid and dashed red lines. In other words, without a field-dependent critical current density, the flux avalanches are always triggered at the indentation. For a field-dependent critical current density $J_{c}(B)$, i.e. with a finite $B_{0} / B_{f}$ ratio, the current density at the indentation (solid red line) decreases faster than that away from the indentation (solid blue line). For large values of $B_{0} / B_{f}$ ratio the first flux avalanche is still triggered at the indentation. However, for weak $B_{0} / B_{f}$ ratios the blue solid line reaches the threshold value first, which means that the first flux avalanche will be triggered away from the indentation. Furthermore, the red solid lines with small $B_{0} / B_{f}$ show two possible situations. For the rightmost red curve, avalanches can still be triggered at the indentation, but the threshold is reached first at the border. In contrast to that, for the leftmost curve, the system is stable near the indentation, where no avalanche can occur.

To pursue our study, we assume a field-dependent sheet current density with $B_{0} / B_{f}=1$ and investigate the influence of the size of the indentation and the ramp rate. According to Ref. [8], where the penetration of magnetic flux through a semicircular edge defect was studied, the excess depth $\Delta$ increases with the size of the indentation. Figure 5 shows the penetration of magnetic flux in superconducting films with indentation of size $2 s \times s$
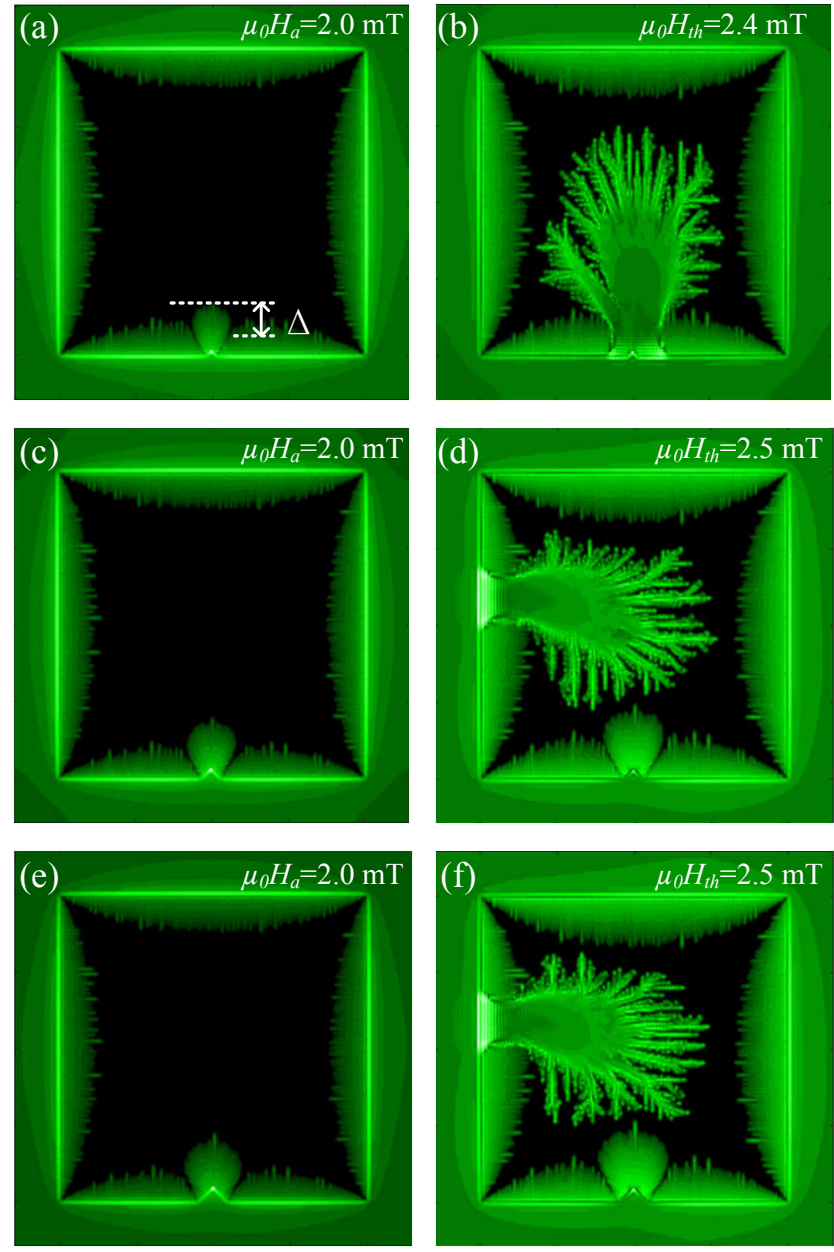

FIG. 5. Simulated distributions of $B_{z}$ just before (left colum$\mathrm{n})$ and after (right column) flux avalanches has been triggered for indentation size $s \times 2 s$ with $s=31 \mu \mathrm{m}$ (a, b), $62 \mu \mathrm{m}$ (c, $\mathrm{d})$, and $93 \mu \mathrm{m}(\mathrm{e}, \mathrm{f})$. In all cases, the substrate temperature is $T_{0}=2 \mathrm{~K}$ and the ramp rate is $\mu_{0} \dot{H}_{a}=1 \mathrm{~T} / \mathrm{s}$.

for three different values of $s: 31 \mu \mathrm{m}(\mathrm{a}, \mathrm{b}), 62 \mu \mathrm{m}(\mathrm{c}$, $\mathrm{d})$, and $93 \mu \mathrm{m}(\mathrm{e}, \mathrm{f})$. Panels on the left column (a, c, e) show the field landscape just before an avalanche has triggered, whereas panels on the right column (b, d, f) correspond to the situation just after a flux burst. We confirm that the excess depth $\Delta$ generated near the indentation is indeed smaller for the smaller defect. Interestingly, for the sample with the smallest triangular indentation, an avalanche is triggered at the defect (Fig. 5(b)) whereas for the two largest indentations, in Fig. 5(d) and Fig. 5(f), the first avalanches are triggered away from the defect. In such cases, the nucleation point is not at the center of the edge, due to the fluctuations in the electric field caused by the quenched disorder, in accordance with the findings of Refs. [22, 32, 33].

It is well known that the characteristics of flux avalanches strongly depend on the environment temperature [17]. The influence of $T_{0}$ in the investigated superconducting film with an indentation is shown in Fig- 

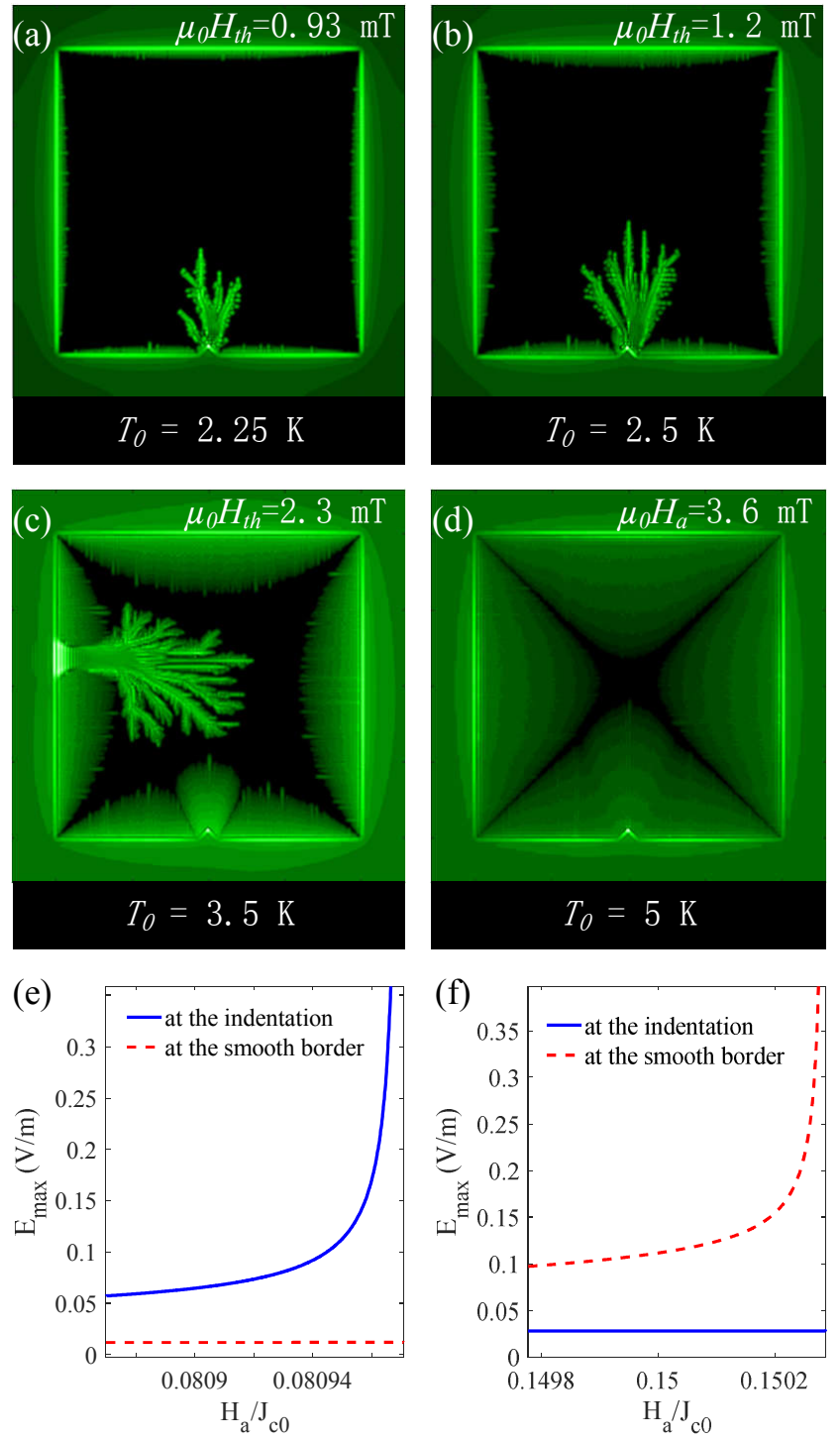

FIG. 6. Distribution of $B_{z}$ as a function of temperature in a sample with an indentation of size $2 s \times s$ with $s=62 \mu \mathrm{m}$ and for $\mu_{0} \dot{H}_{a}=4 \mathrm{~T} / \mathrm{s}$ : (a) $2.25 \mathrm{~K}$ and $\mu_{0} H_{a}=0.93 \mathrm{mT}$, (b) $2.5 \mathrm{~K}$ and $\mu_{0} H_{a}=1.2 \mathrm{mT}$, (c) $3.5 \mathrm{~K}$ and $\mu_{0} H_{a}=2.3 \mathrm{mT}$, (d) $5 \mathrm{~K}$ and $\mu_{0} H_{a}=3.6 \mathrm{mT}$. (e) and (f) show the maximum electric field $E_{\max }$ at the indentation (solid lines) and along the border (dashed lines) for $T_{0}=2.5 \mathrm{~K}$ and $3.5 \mathrm{~K}$ just before the nucleation of the flux avalanches in (b) and (c).

ures $6(\mathrm{a}-\mathrm{d})$ for an indentation with $s=62 \mu \mathrm{m}$, ramp rate $\mu_{0} \dot{H}_{a}=4 \mathrm{~T} / \mathrm{s}$, and several substrate temperature $T_{0}=2.25 \mathrm{~K}(\mathrm{a}), 2.5 \mathrm{~K}(\mathrm{~b}), 3.5 \mathrm{~K}(\mathrm{c})$ and $5 \mathrm{~K}(\mathrm{~d})$. For the lower temperatures (Fig. 6(a, b, c)) the images correspond to an applied field equal to the threshold field for the first avalanche. With increasing temperature, the avalanches become larger, and for a fixed field $H_{a}>H_{t h}$, the number of avalanches is found to decrease due to the reduction of the critical sheet current $J_{c}$ (not shown). The first dendritic flux avalanche is no longer triggered at the indentation when the temperature reaches $3.5 \mathrm{~K}$.

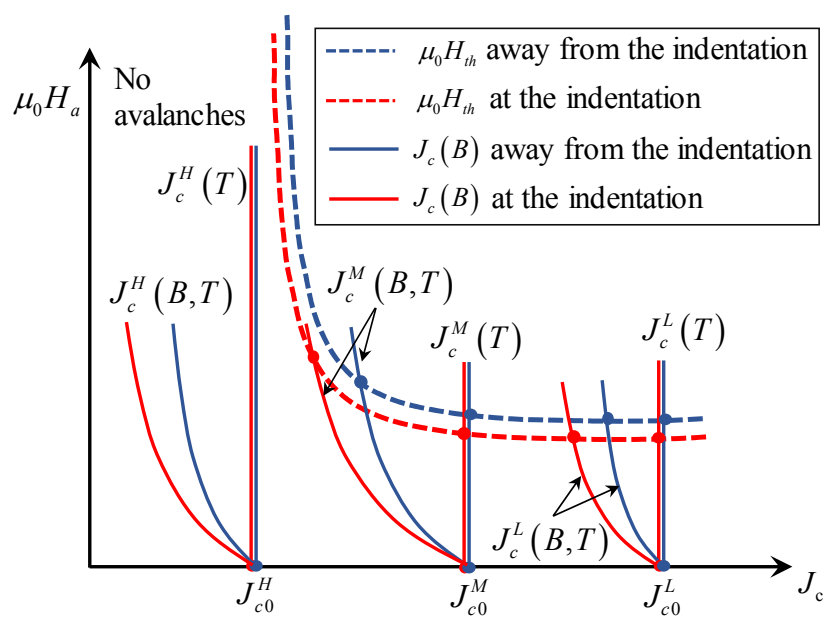

FIG. 7. Generic curves giving the threshold fields $\mu_{0} H_{\text {th }}$ away from the indentation and at the indentation as a function of $J_{c}$. Several cases of $J_{c}(B, T)$ laws with different $T_{0}$ are shown. $J_{c 0}$ with the superscripts $L, M$, and $H$ denote the critical current density at low working temperature, moderate temperature and high temperature in zero magnetic field, respectively.

One can also note that for the highest temperature of 5 $\mathrm{K}$, the flux front almost reaches the center of the sample without any thermomagnetic instability.

Since avalanches are triggered once a threshold electric field is surpassed, it is interesting to analyze the behavior of the electric field in the vicinity of the indentation and along the smooth borders. To that end, we compute the maximum electric field in a square region of size similar to the base of the triangular indentation and including the indentation, and separately we compute the maximum electric field outside this region, i.e. counting the smooth borders. Figures 6(e) and 6(f) show the results of the maximum electric field $E_{\max }$ around the indentation tip (solid lines) and along the smooth border (dashed lines) for $T_{0}=2.5 \mathrm{~K}$ and $3.5 \mathrm{~K}$ right before the first avalanches are triggered. The associated snapshots of magnetic field landscape at the threshold field (i.e. after the avalanche has been triggered) corresponding to panels (e) and (f), are shown in panels (b) and (c), respectively. During an initial phase of increasing applied field and irrespective of the location of avalanche nucleation, the electric field at the tip of the indentation is larger than at any place along the border. However, by further increasing the applied magnetic field, the local temperature of the sample can increase rapidly due to the random perturbations and even exceed the local temperature at the indentation. Under these circumstances, the electric field reaches the threshold conditions for thermomagnetic instability. When the first avalanche nucleates at the indentation, $E_{\max }$ increases rapidly from 0.059 $\mathrm{V} / \mathrm{m}$, while $E_{\max }$ along the border remains constant at about $0.01 \mathrm{~V} / \mathrm{m}$. On the contrary, for the case when the flux avalanche is triggered at the border, there is a rapid 

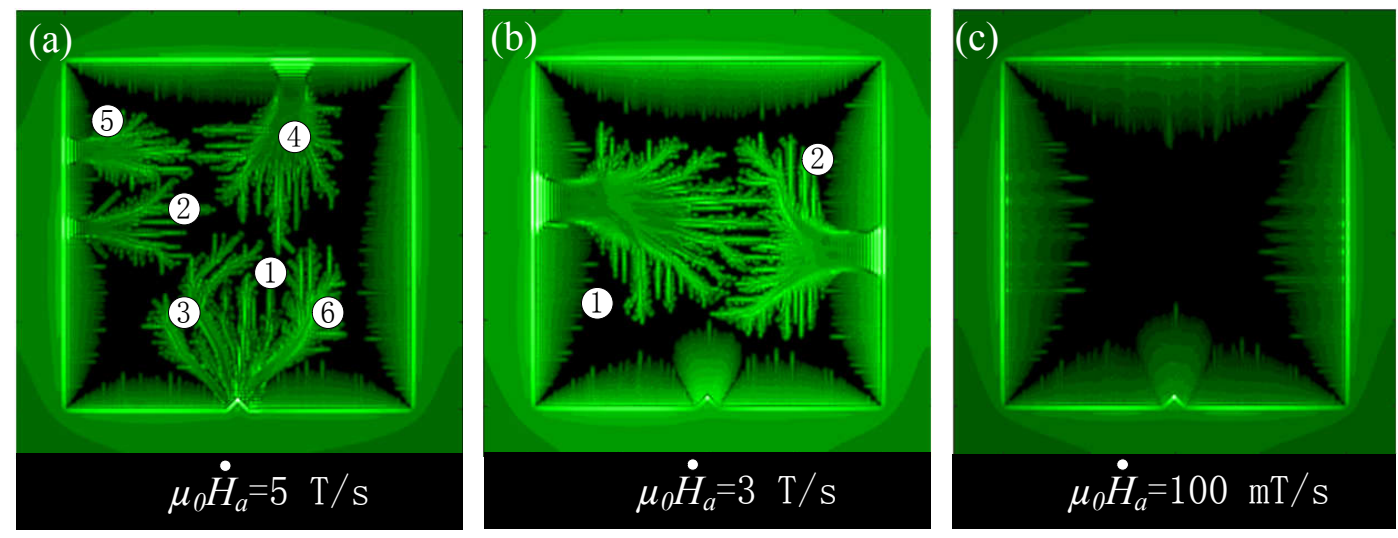

FIG. 8. Distribution of $B_{z}$ as a function of the ramp rate of the applied field $\mu_{0} \dot{H}_{a}$ for $T_{0}=2.5 \mathrm{~K}$ and $\mu_{0} H_{a}=2.8 \mathrm{mT}$. The indentation size is $2 s \times s$ with $s=62 \mu \mathrm{m}$. The numbering within circles indicates the chronological order of flux avalanche appearances.

rise of $E_{\max }$ as $\mu_{0} H_{a}$ gets closer to $H_{\mathrm{th}}$, while the electric field at the tip of the indentation stays constant at about $0.03 \mathrm{~V} / \mathrm{m}$.

In order to better grasp the effect of working temperature on the location of the first flux avalanche, we proceed further with the discussion associated to Fig. 4. To that end, we present in Fig. 7 the situation corresponding to changes in the working temperature $T_{0}$. Similarly as in Fig. 4, the red and blue solid lines represent the $J_{c}\left(H_{a}\right)$ model whereas the $J_{c 0}$ with superscripts $L, M$, and $H$ denote the critical current density at low, moderate, and high working temperatures in zero magnetic field, respectively. At low working temperatures, the avalanches always take place first at the indentation, consistent with the observation in Figures 6(a,b). As the working temperature increases to a moderate value (see $J_{c}^{M}$ ), all the $J_{c}$ curves are shifted to the left. In this case, the shift of curves leads to a reversal of the threshold applied magnetic fields. In other words, it allows the blue solid line to reach its threshold value at lower applied fields than the red solid line and thus the first avalanche is triggered at the smooth border, as shown in Fig. 6(c). At high working temperatures, all the curves are shifted to the stability region, which means that no avalanche can be triggered in the film irrespective of the field-dependent critical current law (see Fig. 6(d)). Note that the above description implicitly assumes that the dashed red and blue lines are temperature independent. Actually, these curves shift to higher $J_{c}$ values as $T_{0}$ increases, and therefore the overall argument advanced above remains valid.

In addition to the substrate temperature, the ramp rate of the applied field $\dot{H}_{a}$ also plays an important role in generating flux instabilities $[9,10,20,34]$. Figure 8 represents the magnetic field distributions of films with an indentation with $s=62 \mu \mathrm{m}$ at $2.5 \mathrm{~K}$, after the applied field increases from zero to $2.8 \mathrm{mT}$, with ramp rates ranging from $100 \mathrm{mT} / \mathrm{s}$ to $5 \mathrm{~T} / \mathrm{s}$. For the highest ramp rate (Fig. 8(a)) many dendritic avalanches are triggered. The large multibranched avalanche emerging from the indentation is composed of three separated avalanches occuring at different magnetic fields. For a ramp rate of $3 \mathrm{~T} / \mathrm{s}$ (Fig. 8(b)) one sees that the number of avalanches decreases and the avalanche morphology evolves to larger trunks. Remarkably, the two dendritic flux avalanches now occur along the borders. For even smaller ramp rates (Fig. 8(c)) the field penetration is smooth and the thermomagnetic instability condition is not reached.

In order to clarify the process of nucleation of flux avalanches, we consider the magnetic moment in units of $m_{0}=a^{3} j_{c 0} d$ and the maximum local temperature as a function of the applied field $H_{a} / j_{c 0} d$ for different substrate temperatures $T_{0}$, as shown in Fig. 9. For $T_{0}=2.25 \mathrm{~K}$, the magnetic moment shows frequent flux jumps of small amplitudes. As mentioned above, the first of these jumps corresponds to an avalanche triggered at the indentation. As the applied field increases, subsequent jumps correspond to flux avalanches avoiding each other until a new avalanche will emerge from the indentation tip again. For $T_{0}=2.5 \mathrm{~K}$, the first jump corresponds to a flux avalanche nucleated at the indentation tip. For the two highest temperatures $\left(T_{0}=2.75 \mathrm{~K}\right.$ and $\left.T_{0}=3 \mathrm{~K}\right)$, no avalanche nucleate at the indentation tip but rather away from it for the field ramp rate here investigated. In this case, the magnetic moment curve shows less frequent flux jumps of larger size and triggering at larger threshold fields. It thus appears that the avalanches triggered at the border at the highest temperatures are larger and less numerous than those initiated at the indentation at lower temperatures (see also Fig. 6). Figure 9 (b) shows the temperature differences $\Delta T$, between the substrate temperature $T_{0}$ and the maximum local temperature $T_{\max }$, as a function of $H_{a} / j_{c 0} d$. The main panel corresponds to the two highest temperatures $T_{0}$, for which the first flux avalanches are triggered along the border, while the inset shows the case of the two lowest temperatures for which the first avalanches nucleate at the indention tip. It can be seen that every spike in the temperature $\Delta T$ corresponds to a jump in magnet- 

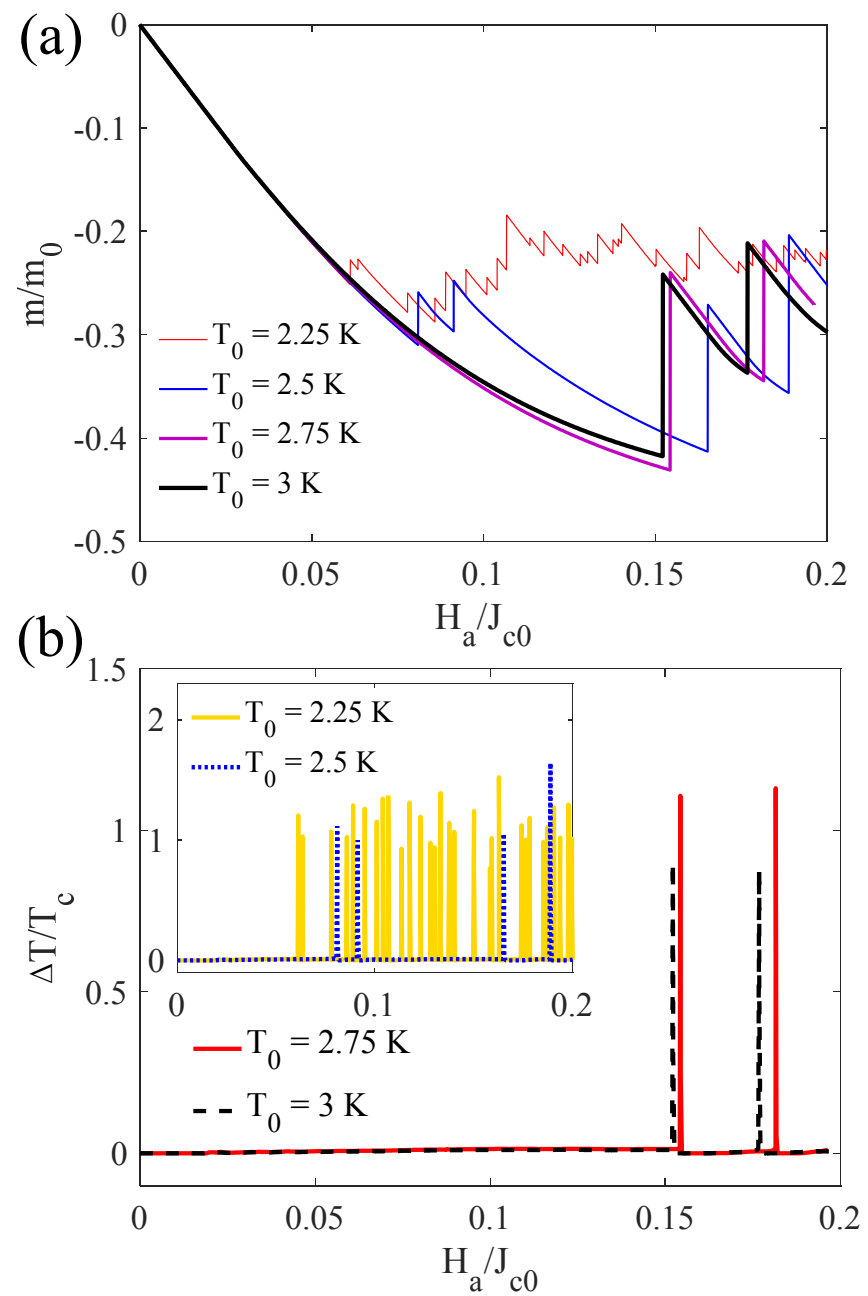

FIG. 9. (a) Magnetic moment in units of $m_{0}=a^{3} j_{c 0} d$ and (b) maximum temperature of the superconducting film with an edge indentation (with $s=62 \mu \mathrm{m}$ ) as a function of the applied field at four different $T_{0}$ with $\mu_{0} \dot{H}_{a}=4 \mathrm{~T} / \mathrm{s}$.

ic moment plotted in Fig. 9(a). Note that the $m\left(H_{a}\right)$ curves for $T_{0}=2.75 \mathrm{~K}$ and $3 \mathrm{~K}$, exhibiting two jumps corresponding to avalanches away from the indentation, are rather similar. This observation suggests that in this case the substrate temperature $T_{0}$ has little effect on the magnetic moment and the maximum temperature. Let us now investigate the impact of the ramp rate on magnetization. Figure 10 shows the magnetization curves and the maximum local temperatures for a substrate temperature $T_{0}=2.5 \mathrm{~K}$ and different ramp rates, $\mu_{0} \dot{H}_{a}=5 \mathrm{~T} / \mathrm{s}$, $4 \mathrm{~T} / \mathrm{s}, 3 \mathrm{~T} / \mathrm{s}$ and $1 \mathrm{~T} / \mathrm{s}$. The inset of Fig. 10(b) presents the maximum temperatures at $\mu_{0} \dot{H}_{a}=5 \mathrm{~T} / \mathrm{s}$ and $4 \mathrm{~T} / \mathrm{s}$, which corresponds to the case of flux avalanches triggered at the indentation. It can be found that both the size and the frequency of the jumps in the normalized magnetization and the maximum temperature depend on the ramp rate. The jump frequency increases with the ramp rate, while the jump size decreases with it. The ramp rate, however, has little influence on the jump size and
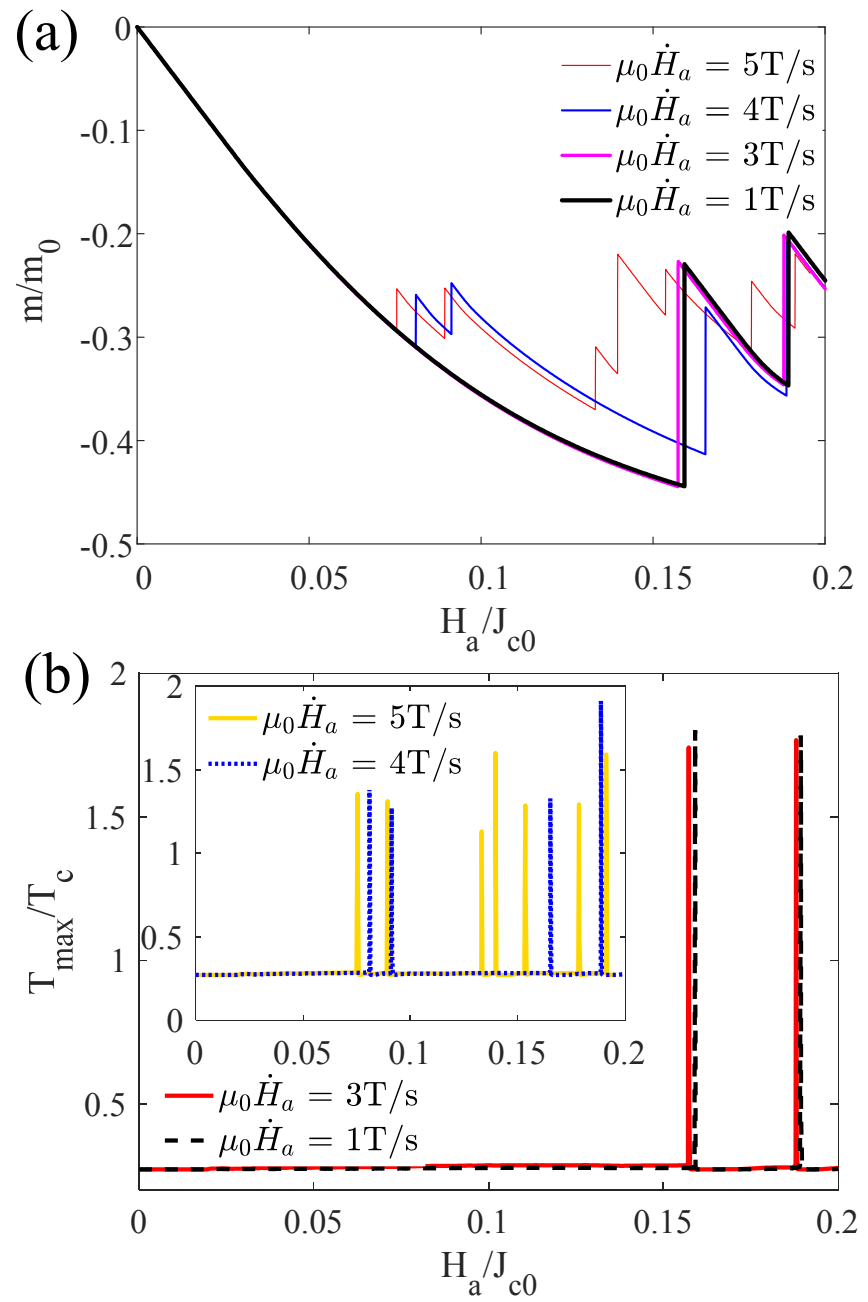

FIG. 10. (a) Magnetic moment in units of $m_{0}=a^{3} j_{c 0} d$ and (b) maximum temperature of the superconducting film with an edge indentation (with $s=62 \mu \mathrm{m}$ ) as a function of the applied field at four different $\mu_{0} \dot{H}_{a}$ at $T_{0}=2.5 \mathrm{~K}$.

frequency when avalanches occur at the border, similar to the insensitivity to the substrate temperature pointed out above. In addition, we find that the threshold magnetic field is smaller if the ramp rate is larger. This result is in agreement with the theory in Ref. [35], which states that $H_{t h}=d j_{c} / \pi \operatorname{atanh}\left(h T^{*} / n a d j_{c} \mu_{0} \dot{H}_{a}\right)$, where $T^{*}=\left|\partial \ln j_{c} / \partial T\right|^{-1}$.

The discussion above suggests that the nucleation locus for flux avalanches depends on both the ramp rate of the applied magnetic field and the temperature $T_{0}$. Therefore, we map the different nucleation locations obtained from the numerical simulations in the $\mu_{0} \dot{H}_{a}-T_{0}$ planes with field-independent critical current $J_{c}(T)$ (see Fig. 11(a)) and field-dependent $J_{c}(B, T)$ (see Fig. 11(b)). There are only two regions in Fig. 11(a), i.e., flux avalanches triggered preferentially at the indentation (the blue region) and smooth penetration(the green region). This result is consistent with the predictions of Fig. 4 and above explanations with field-independent $J_{c}$. 
In Fig. 11(b), the lower blue regime corresponds to the lowest values of $T_{0}$ and largest values of $\mu_{0} \dot{H}_{a}$, for which magnetic flux avalanches were found to nucleate at the indentation. The middle yellow region corresponds to intermediate temperatures for which flux avalanches are preferentially nucleated along the smooth borders, away from the indentation. Note that for a fixed $T_{0}$, the lowest ramp rates are seen to favor nucleation away from the indentation, whereas flux avalanches nucleate at the indentation for higher ramping rates. This is likely due to an increase of the electric field at the tip by current bending. For the green region extending to the highest temperatures, no flux avalanches are observed and the flux penetration is smooth. This regime corresponds to lower sheet current densities and locally lower electric fields yielding small Joule heating, so that no thermomagnetic instability is triggered. The results of the three temperature zones corresponding to a fixed ramp rate are consistent with the analysis in Fig. 7.

\section{CONCLUSIONS}

In summary, we have used numerical simulations with field-dependent critical current to investigate how a triangular edge indentation affects the flux penetration in a superconducting film. By comparing numerical result$\mathrm{s}$ obtained from field-dependent and field-independent critical current, we find that the excess flux penetration $\Delta$ obtained from the field-dependent $J_{c}$ model is larger than that from the field-independent $J_{c}$ model and it decreases with increasing $B_{0}$. Namely, the indentation helps magnetic flux to gradually enter and so to avoid thermomagnetic instabilities for small $B_{0}$, which corresponds to a strong field-dependent critical sheet current density $J_{c}$. Our numerical results show that $\Delta$ increases with the size of the triangle indentation, consistent with previous reports [8]. However, against the common wisdom, the larger indentation induces no flux avalanches due to a reduction of $J_{c}$, easing the entrance of magnetic flux. Therefore, the field-dependent $J_{c}(B, T)$ model not only implies a decrease of the local current density, but also increases the threshold magnetic fields for the first flux avalanche triggered at the indentation. So, it is the competition between which $J_{c}$ curve $\left(J_{c}\right.$ curve at the indentation or $J_{c}$ curve away from indentation) first reaches its critical value to trigger a flux avalanche. The fast-rising feature of threshold magnetic field $\left(H_{t h}\left(J_{c}\right)\right.$ curves) provides the opportunity for the observed first flux avalanche triggered away from indentation. By varying the temperature with different field ramp rates, we build up the phase diagrams in the $\mu_{0} \dot{H}_{a}-T_{0}$ planes delineating the boundaries of the regimes of smooth penetration, thermomagnetic instabilities nucleated at the smooth border and avalanches triggered at the indentation. An explanation to the previous experimental results has been proposed in terms of the ability of edge indentation to promote the flux entry, which decreases
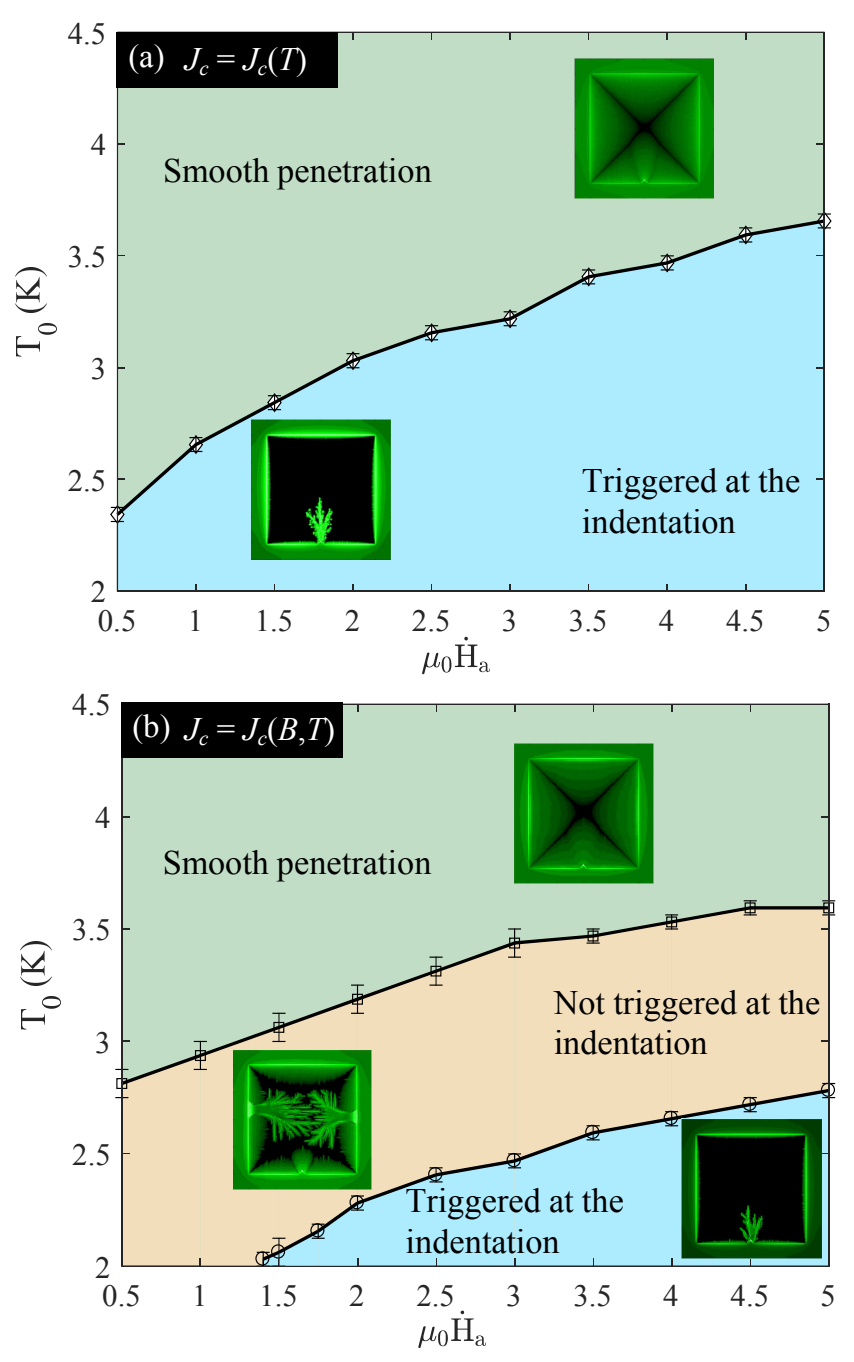

FIG. 11. Thermomagnetic stability diagram in the $\mu_{0} \dot{H}_{a}-T_{0}$ planes with $J_{c}=J_{c}(T)$ and $J_{c}=J_{c}(B, T)$ for a superconducting film with an edge triangular indentation with $s=62$ $\mu \mathrm{m}$. Green, yellow, and blue denote the regions of smooth penetration, with avalanches nucleating at the border, and avalanches nucleating at the indentation, respectively. The error bars show the accuracy of the dividing lines.

the local $J_{c}$, weakens the current crowding and increases the threshold applied magnetic field at the indentation. Within the region where avalanches are triggered at the indentation, the current bending effect at the indentation plays a dominant role at low temperature or high ramp rate. We stress on the fact that for the case of field-independent $J_{c}$ model, magnetic flux avalanches are always triggered at the indentation. Further refinements of the present model could be done by taking into accoun$\mathrm{t}$ surface barriers effects. It would be interesting to extend and confront the present investigation to the case of current induced flux avalanches caused by flux focussing effect as experimentally addressed in Ref. [36, 37] 
Acknowledgement We acknowledge support by the National Natural Science Foundation of China (Grant Nos. 11972298 and 11702218), Natural Science Basic Research Plan in Shaanxi Province of China (Grant No. 2018JM1003), Young Talent Fund of University Associa- tion for Science and Technology in Shaanxi, China (Grant No. 20180501), and the Fundamental Research Funds for the Central Universities (Grant No. 300102129104).
[1] A. Gurevich, and M. Friesen, Phys. Rev. B 62, 4004 (2000); ibid. Phys. Rev. B 63, 064521 (2001).

[2] A. L. Rakhmanov, D. V. Shantsev, Y. M. Galperin and T. H. Johansen, Phys. Rev. B 70, 224502 (2004).

[3] I. S. Aranson, A. Gurevich, M. S. Welling, R. J. Wijngaarden, V. K. Vlasko-Vlasov, V. M. Vinokur and U. Welp, Phys. Rev. Lett. 94, 037002 (2005).

[4] D. V. Denisov, D. V. Shantsev, Y. M. Galperin, E.-M. Choi, H.-S. Lee, S.-I. Lee, A. V. Bobyl, P. E. Goa, A. A. F. Olsen and T. H. Johansen, Phys. Rev. Lett. 97, 077002 (2006).

[5] J. R. Clem, and K. K. Berggren, Phys. Rev. B 84, 174510 (2011).

[6] Th. Schuster, H. Kuhn and E. H. Brandt, Phys. Rev. B 54, 3514 (1996).

[7] Th. Schuster, M. V. Indenbom, M. R. Koblischka, H. Kuhn and H. Kronmüller, Phys. Rev. B 49, 3443 (1994).

[8] J. I. Vestgården, D. V. Shantsev, Y. M. Galperin and T. H. Johansen, Phys. Rev. B 76, 174509 (2007).

[9] Z. Jing, H. D. Yong and Y. H. Zhou, Supercond. Sci. Technol. 28, 075012 (2015).

[10] M. Baziljevich, E. Baruch-El, T. H. Johansen and Y. Yeshurun, Appl. Phys. Lett. 105, 012602 (2014).

[11] J. Brisbois, O.-A. Adami, J. I. Avila, M. Motta, W. A. Ortiz, N. D. Nguyen, P. Vanderbemden, B. Vanderheyden, R. B. G. Kramer and A. V. Silhanek, Phys. Rev. B 93, 054521 (2016).

[12] G. Shaw et al. Rev. Sci. Instrum. 89, 023705 (2018).

[13] M. R. Koblischka and R. J. Wijngaarden, Supercond. Sci. Technol. 8, 199 (1995).

[14] C. Jooss, J. Albrecht, H. Kuhn, S. Leonhardt and H. Kronmüller, Rep. Prog. Phys. 65, 651 (2002).

[15] C. Liu, X. Y. Zhang and Y. H. Zhou, Meas. Sci. Technol. 30, 115904 (2019).

[16] C. A. Durán, P. L. Gammel, R. E. Miller and D. J. Bishop, Phys. Rev. B 52, 75 (1995).

[17] T. H. Johansen, M. Baziljevich, D. V. Shantsev, P. E. Goa, Y. M. Galperin, W. N. Kang, H. J. Kim, E. M. Choi, M.-S. Kim and S. I. Lee, Europhys. Lett. 59, 599 (2002).

[18] J. Albrecht, A. T. Matveev, M. Djupmyr and G. Schütz, Appl. Phys. Lett. 87, 182501 (2005).

[19] P. Leiderer, J. Boneberg, P. Brüll, V. Bujok and S. Her- minghaus, Phys. Rev. Lett. 71, 2646 (1993).

[20] E. Baruch-El, M. Baziljevich, B. Ya. Shapiro, T. H. Johansen, A. Shaulov and Y. Yeshurun, Phys. Rev. B 94, 054509 (2016).

[21] J. Brisbois et al, Phys. Rev. B 95, 094506 (2017).

[22] J. I. Vestgården, D. V. Shantsev, Y. M. Galperin and T. H. Johansen, Phys. Rev. B 84, 054537 (2011).

[23] Z. Jing, H. D. Yong and Y. H. Zhou, Supercond. Sci. Technol. 29, 105001 (2016).

[24] J. McDonald and J. R. Clem, Phys. Rev. B 53, 8643 (1996).

[25] C. Xue, A. He, H. D. Yong and Y. H. Zhou, AIP Adv. 3, 122110 (2013).

[26] E. H. Brandt, Phys. Rev. B 52, 15442 (1995).

[27] J. I. Vestgården, P. Mikheenko, Y. M. Galperin and T. H. Johansen, New J. Phys. 15, 093001 (2013).

[28] M. Motta et al, Phys. Rev. B, 89, 134508 (2014).

[29] J. I. Vestgården, F. Colauto, A. M. H. de Andrade, A. A. M. Oliveira, W. A. Ortiz and T. H. Johansen, Phys. Rev. B 92, 144510 (2015).

[30] Z, Jing, H. D. Yong and Y. H. Zhou, J. Appl. Phys. 121, 023902 (2017).

[31] D. V. Denisov, A. L. Rakhmanov, D. V. Shantsev, Y. M. Galperin and T. H. Johansen, Phys. Rev. B, 73, 014512 (2006).

[32] J. I. Vestgården, Y. M. Galperin and T. H. Johansen, Physica C, 479, 92 (2012).

[33] V. V. Yurchenko, D. V. Shantsev, T. H. Johansen, M. R. Nevala, I. J. Maasilta, K. Senapati and R. C. Budhani, Phys. Rev. B 76, 092504 (2007).

[34] D. Carmo, F. Colauto, A. M. H. de Andrade, A. A. M. Oliveira, W. A. Ortiz and T. H. Johansen, IEEE Trans. Appl. Supercond. 25, 7501004 (2015).

[35] J. I. Vestgården, Y. M. Galperin and T. H. Johansen, Phys. Rev. B 93, 174511, (2016).

[36] D. Carmo, F. Colauto, A. M. H. de Andrade, A. A. M. Oliveira, W. A. Ortiz, Y. M. Galperin and T. H. Johansen, Supercond. Sci. Technol. 31, 115009 (2018)

[37] D. Carmo, F. Colauto, A. M. H. de Andrade, A. A. M. Oliveira, W. A. Ortiz and T. H. Johansen, Supercond. Sci. Technol. 29, 095003, (2016). 